\title{
Biological effects of a new set 1,2,4-triazolo[1,5-a] quinazolines on heart rate and blood pressure
}

\author{
Rashad Al-Salahi', Kamal-Eldin El-Tahir ${ }^{2}$, Ibrahim Alswaidan' ${ }^{1}$ Nabih Lolak ${ }^{3}$, Mohammed Hamidaddin ${ }^{1}$ \\ and Mohamed Marzouk ${ }^{1 *}$
}

\begin{abstract}
Background: Several quinazoline and triazole derivatives are reported to possess a wide-range of interesting pharmacological effects. Although various triazoloquinazoline subclasses having been synthesized and studied, the preparation of 1,2,4-triazolo[1,5-a]quinazolines as antihypertensive agent is still relatively unexplored. In continuation of our earlier research, we aimed at the synthesis and development of various potent antihypertensive 1,2,4-triazoloquinazoline derivatives.

Results: A new series of 1,2,4-triazolo[1,5-a]quinazoline derivatives have been synthesized. Their structures were mainly established by spectroscopic methods of analysis (IR, MS, ${ }^{1} \mathrm{H}$ and ${ }^{13} \mathrm{C}$ NMR). Their in vivo antihypertensive activity was evaluated by tail cuff method using Muromachi Blood Pressure Monitor (Model MK 2000) for rats and mice. Some of the tested compounds were found to exhibit valuable effects in terms of heart rate and blood pressure. According to the biological results, some of tested derivatives have abolished completely the tachycardia of the parent compounds and may be studied and modified as potential adrenoblockers and cardiac stimulant.

Conclusion: New series of fifteen 1,2,4-triazolo[1,5-a]quinazolines were synthesized by convenient methodology from four key molecules, whereby their structures were established by advanced spectroscopic analyses. Some lead compounds have abolished completely the tachycardia of the parent compounds, that may be examined as potent adrenoblockers and some other compounds seem to be a cardiac stimulant or may be modified to enhance their hypotensive activity.
\end{abstract}

Keywords: 1,2,4-Triazolo[1,5-a]quinazoline, Heart rate, Antihypertensive activity

\section{Background}

Arterial diseases cause more premature deaths than all other disorders such as cancer and infections combined. High blood pressure has been identified as the most powerful one among the major risk factors for arterial diseases [1]. Now-a-days, several efforts have been made in search of potent anti-hypertensive drugs because hypertension was commonly proved to cause heart failure. Quinazolines and their condensed products are reported to possess several interesting pharmacological effects such as antihypertensive [2], antihistaminic [3,4], analgesic, anti-inflammatory [5,6], anticancer [7], and anti-HIV [8] activities. Prazocin, terazocin and doxazocin as quinazoline derived $\alpha-1$ blockers, are reputed class of antihypertensive

\footnotetext{
* Correspondence: msmarzouk@yahoo.co.uk

'Department of Pharmacetical Chemistry, College of Pharmacy, King Saud

University, P. O. Box 2457, Riyadh 11451, Saudi Arabia

Full list of author information is available at the end of the article
}

agents. In spite of various triazoloquinazoline systems having been synthesized and studied, the elaborating of 1,2,4-triazolo[1,5-a]quinazoline as antihypertensive agent is still relatively unexplored [1]. Moreover, Some of potent antihypertensive 1,2,4-triazoloquinazoline derivatives were reported [1]. In view of these facts and continuation to our earlier reported triazoloquinazolines researches [1,9-12], we aimed at synthesis of various substituted 1,2,4-triazolo [1,5-a]quinazolines and evaluation their biological effects on heart rate and blood pressure.

\section{Results and discussion}

Synthetic chemistry

As outlined in Scheme 1, the target molecules were synthesized by starting with the preparation of our key materials (1-4) according to the literature [13-15]. The structures of $\mathbf{1}-\mathbf{3}$ were characterized by NMR, MS, IR spectra and compound $\mathbf{1}$ has been unambiguously 
<smiles>PSc1nc2nc(Oc3ccccc3)nn2c2ccccc12</smiles>

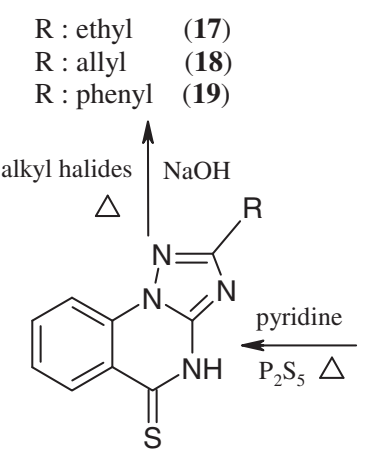

$\mathrm{R}: \mathrm{O}-\mathrm{Ph} \quad$ (15) $\mathrm{R}: \mathrm{S}^{-} \mathrm{CH}_{3}(\mathbf{1 6})$<smiles>[R]c1nc2nc(Cl)c3ccccc3n2n1</smiles>

$\mathrm{R}: \mathrm{O}-\mathrm{Ph} \quad$ (11) $\mathrm{R}: \mathrm{S}^{-\mathrm{CH}_{3}}$ (12) $\mathrm{R}: \mathrm{SO}_{2} \mathrm{CH}_{3}$ (13)<smiles>[R]c1ccc2c(=O)[nH]c3nc([R])nn3c2c1</smiles>

$$
\mathrm{R}: \mathrm{O}-\mathrm{Ph}, \mathrm{R}^{1}: \mathrm{H}
$$
$\mathrm{R}: \mathrm{S}-\mathrm{CH}_{3}, \mathrm{R}^{1}: \mathrm{H}$ $\mathrm{R}: \mathrm{O}-\mathrm{Ph}, \mathrm{R}^{1}: \mathrm{CH}_{3}$ $\mathrm{R}: \mathrm{SO}_{2}-\mathrm{CH}_{3} \mathrm{R}^{1}: \mathrm{H}$

alkyl halides/RT $\mathrm{K}_{2} \mathrm{CO}_{3} / \mathrm{DMF}$

(4)<smiles>Pc1nc2nc(Oc3ccccc3)nn2c2ccccc12</smiles>

R : ethoxyl (14)<smiles>[R]c1nc2n([R])c(=O)c3ccccc3n2n1</smiles>

$\mathrm{R}: \mathrm{O}-\mathrm{Ph}, \quad \mathrm{R}^{1}: p-\mathrm{NO}_{2}-\mathrm{Bn}(\mathbf{5})$

$\mathrm{R}: \mathrm{O}-\mathrm{Ph} \quad \mathrm{R}^{1}$ : ethyl

$\mathrm{R}: \mathrm{O}-\mathrm{Ph} \quad \mathrm{R}^{1}$ : allyl

$\mathrm{R}$ : $\mathrm{SO}_{2} \mathrm{CH}_{3} \mathrm{R}^{1}$ : ethyl

$\mathrm{R}: \mathrm{SO}_{2} \mathrm{CH}_{3} \mathrm{R}^{1}$ : allyl

Scheme 1 Synthesis of $[1,2,4]$ triazolo[1,5-a]quinazoline derivatives.

proven by X-ray crystallography [13-18]. Regioselective $N$-alkylation has been well documented in the literature $[13,14]$. Accordingly, when the triazoloquinazolin-5-ones $(\mathbf{1}, \mathbf{4})$ were allowed to react with alkyl halides in a molar ratio of 1:1.5 in dry dimethyl formamide at room temperature in the presence of potassium carbonate, the corresponding 4-alkyl[1,2,4] triazolo[1,5-a]quinazolin-5ones $(\mathbf{5}-\mathbf{1 0})$ resulted in $71-87 \%$ yield. The products $\mathbf{5 - 1 0}$ were obtained as coloress solid and their IR spectra displayed a strong $(\mathrm{C}=\mathrm{O})$ absorption band in the range of $1675-1689 \mathrm{~cm}^{-1}$. When equimolar amounts of triazoloquinazolin-5-one $\mathbf{1}$ and phosphorus pentasulfide were allowed to react in dry pyridine under reflux for $3 \mathrm{~h}$, the desired triazoloquinazolin-5-thione (15) could be isolated as yellow solid in excellent yield of 92\% [18]. The IR spectra of compound $\mathbf{1 5}$ displayed a weak $(\mathrm{C}=\mathrm{S})$ absorption band at around $1197 \mathrm{~cm}^{-1}$ and characterized by NMR and MS spectra. The structure of compound 16 has been previously proved by NMR, MS and X-ray $[14,17]$. Treatment of the $[1,2,4]$ triazoloquinazolin-5-thione $\mathbf{1 5}$ with different alkyl halides in aqueous sodium hydroxide solution $(2 \mathrm{M})$ afforded smoothly the expected thioethers (17-19) in 54-75\% yield. Conversion of [1,2,4]triazoloquinazolin-5-ones $(\mathbf{1 , 2 , 4 )}$ into 5-chloro-[1,2,4]triazolo[1,5-a] quinazolines (11-13) have been successfully achieved by phosphorus oxychloride in boiling benzene for $2.5 \mathrm{~h}$, followed by treatment with a saturated aqueous solution of potassium carbonate [19]. The formation of 11-13 was accompanied by the gradual disappearance of the characteristic $(C=O)$ band of 1 and 2 at $1685-1711 \mathrm{~cm}^{-1}$. When compound $\mathbf{1 1}$ was reacted at ambient temperature with sodium ethoxide in ethanol, the triazoloquinazoline (14) could be obtained in $52 \%$ yield. According to literature [20], reaction of compound $\mathbf{2}$ with hydrogen peroxide in boiling glacial acetic acid, followed by treatment with hot water, the corresponding triazolo[1,5-a]quinazolin-5-one (4) was obtained in good yield. IR spectrum of compound 4 is characterized by a strong $(\mathrm{C}=\mathrm{O})$ absorption band at $1699 \mathrm{~cm}^{-1}$. Analogous to the reaction of 2 with hydrogen peroxide, the corresponding compound $\mathbf{1 3}$ was obtained from 12. The MS spectra of $\mathbf{4}$ and $\mathbf{1 3}$ showed molecular ion peaks at $m / z 263$ and $282\left(\mathrm{M}^{*+}, 100 \%\right)$, corresponding to their molecular formulae. As well as the structures of $\mathbf{4}$ and 13 were characterized by NMR, MS, IR spectra, and have been unambiguously proven by $\mathrm{X}$-ray crystallography (Figure 1).

Generally, uncorrected melting points of all compounds were recorded and their chemical structures primarily consistent with their IR and MS data (see experimental section) and confirmed by ${ }^{1} \mathrm{H}$ - and ${ }^{13} \mathrm{C}$ NMR spectroscopy (splitting pattern, $\delta$ - and $J$-values and comparison with literature of structural related compounds). In the tricyclic nucleus, the benzofused moiety was deduced from its own four one proton ${ }^{1} \mathrm{H}$-signals as two $\mathrm{dd}$ (or br d) resonances with $J_{\text {ortho }}(7.5-8.5 \mathrm{~Hz})$ and $J_{\text {meta }}(1-2 \mathrm{~Hz})$ assignable for $\mathrm{H}-9$ and $\mathrm{H}-6$ and two td (or br t) resonances with 

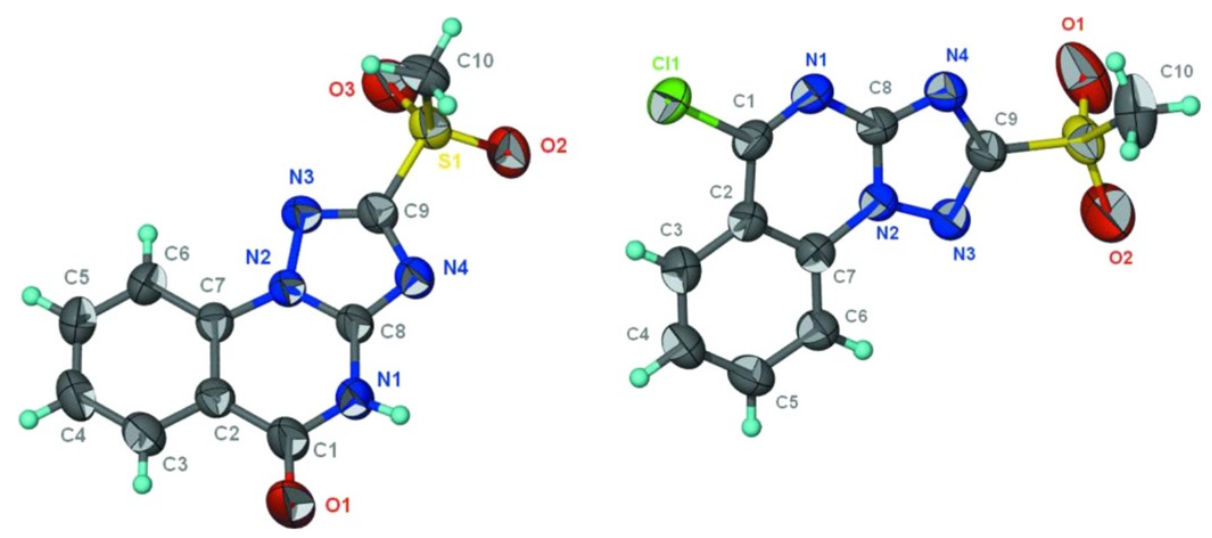

Figure $1 \mathrm{X}$-ray single crystal 3D-structures of compounds 4 and 13.

$J_{\text {ortho }}$ and $J_{\text {meta }}$ for $\mathrm{H}-8$ and $\mathrm{H}-7$, respectively. The exchangeable $\mathrm{NH}$-proton was interpreted in ${ }^{1} \mathrm{H}$ NMR of 3 and $\mathbf{4}$ at $\delta>13 \mathrm{ppm}$ and were absent in all spectra of $\mathrm{N}$-alkyl or 5 -chloro-triazoloquinazolines. ${ }^{13} \mathrm{C}$ NMR spectra proved the main tricyclic moiety through characteristic nine resonances including the most downfield key signal of $\mathrm{C}-2$ assigned at $\approx 165 \mathrm{ppm}$ in 2-phenoxy derivatives (e.g. 3, 5-7) that was observed relatively upfield at $\approx 160$ in case of 2-methylsulfonyl function (e.g. 4, 8-10) due to the stronger $-\mathrm{R}$ and $-\mathrm{I}$ (deshielding) effect of $\mathrm{O}$-phenoxy than $-\mathrm{SO}_{2}$. Another key ${ }^{13} \mathrm{C}$-signal was $\mathrm{C}-5$ that interpreted at $\delta \approx 158-160 \mathrm{ppm}$ in 5 -ones $(3-10), \approx 167$ in 5 - $C l$ derivatives $(\mathbf{1 1}, \mathbf{1 3})$ and $\approx 185$ in case of 5-thione (15). The 2-phenoxy function was easily deduced from its intrinsic three resonances at $\approx 7.46$ (td, $J=8.5,2), 7.33$ (br d, $J=8$ ) and 7.26 (br t, $J=7.5$ ) for $\mathrm{H}-3^{\prime} / 5^{\prime}, \mathrm{H}-2^{\prime} / 6^{\prime}$ and $\mathrm{H}-4^{\prime}(\mathbf{5 , 1 0})$ and confirmed through its four ${ }^{13} \mathrm{C}$ resonances at $\delta \mathrm{ppm} \approx 154,130$, 124 and 119 interpretable for $C-1^{\prime}, 3^{\prime} / 5^{\prime}, 4^{\prime}$, and $2^{\prime} / 6^{\prime}$, respectively. Methylsulfonyl function was concluded from its own ${ }^{1} \mathrm{H}$ and ${ }^{13} \mathrm{C}$ resonances of $\mathrm{O}_{2} \mathrm{~S}-\mathrm{CH}_{3}$ at $\approx 3.45$ (s) and $42 \mathrm{ppm}$, respectively. $N$-alkylation with $p$-nitrobenzyl was unambiguously confirmed by its $\mathrm{A}_{2} \mathrm{M}_{2}$ spin coupling system of the two ortho-doublets at about 8.2 and 7.7 $(J=8.5)$ for $\mathrm{H}-3 " / 5^{\prime \prime}$ and $\mathrm{H}-2 " / 6^{\prime \prime}$ together with the downfield located $\mathrm{CH}_{2}$-singlet at $\approx 5.4$ and its ${ }^{13} \mathrm{C}$-signal at $46 \mathrm{ppm}$ by the deshielding effect of tertiary $N$-atom $(5, \mathbf{1 0})$. Whereas, alkylation with allyl moiety was proved by its four characteristic ${ }^{1} \mathrm{H}$-resonances with intrinsic splitting pattern at about $5.9(\mathrm{~m}), 5.25(\mathrm{dd}, J=17.5,1.5), 5.20$ (dd, $J=10.5,1.5)$ and $4.72(\mathrm{~d}, J=5)$ assignable for olefinic methine and methylene and $\mathrm{CH}_{2}$-saturated types $(7,9)$. The corresponding three ${ }^{13} \mathrm{C}$-resonances were interpreted at $\delta$ ppm 131.2, 117.5 and 45.2 for C-2, C-3 and C- 1 of allyl group, respectively. In compound 18, 5-S-allyl protons were interpreted with the same splitting pattern but highly deshielded at 6.04, 5.42, 5.20 and 4.09 by higher electronegativity of $S$-atom and their ${ }^{13} \mathrm{C}$-signals were reported at $129.6(\mathrm{C}-2 "), 115.1(\mathrm{C}-3 ")$, and 32.1 $(\mathrm{C}-1 ")$. Moreover, $\mathrm{N}$-ethyl function was observed in the form of a typical $\mathrm{A}_{2} \mathrm{X}_{3}$ spin coupling system as a quartet $\left(\mathrm{CH}_{2}\right)$ and triplet $\left(\mathrm{CH}_{3}\right)$ at about $4.2(\mathrm{q}, J=7)$ and $1.3(\mathrm{t}, J=7)$ and confirmed by their corresponding ${ }^{13} \mathrm{C}$-resonances at $\delta$ ppm 38.5 and $12.4(6,8)$. In 17 and $19 S$-ethyl and $S$-phenyl functions showed the same splitting pattern of $N$-ethyl and $O$-phenyl but with slightly different $\delta$-values in both ${ }^{1} \mathrm{H}$ and ${ }^{13} \mathrm{C}$ NMR spectra. All other ${ }^{1} \mathrm{H}$ - and ${ }^{13} \mathrm{C}$-resonances of all structures were finally assigned on the basis of comparison with literature data of structure related compounds and according to application of substitution additive rules of ${ }^{13} \mathrm{C}$ NMR.

\section{Antihypertensive activity}

In vivo antihypertensive activity study of the title compounds 1-19 was performed by tail cuff method using Muromachi Blood Pressure Monitor for rats and mice (Model MK 2000). The obtained results, in Table 1, revealed that the nature of substituent and substitution pattern on the tricyclic systems 1-4 may have had a considerable impact on the heart rate and blood pressure in all synthesized derivatives (1-19).

Basically, the parent $\mathbf{1}$ was found to increase the heart rate, however it has not demonstrated any effect on blood pressure. Replacement of phenoxy group on position $\mathbf{2}$ by a sulfanyl group in case of compound $\mathbf{2}$ has been abolished effect on the heart rate. On the other hand, presence of sulfonyl group in $\mathbf{4}$ was found to induce the bradycardia, however no effect on blood pressure was noticed. Introduction of methyl group on the lead compound $\mathbf{1}$ to afford $\mathbf{3}$ did offer advantageous effect on reduction of blood pressure accomplished by suppressing effect on heart rate in regard to 3 . Alkylation of lactam moiety in $\mathbf{1}$ furnished $N$-alkylated products $5-7$, that did demonstrate remarkable effect. For instance, compound $\mathbf{5}$ was induced bradycardia, however great increasing in the heart rate showed by $\mathbf{6}$, whereas 


\begin{tabular}{|c|c|c|c|}
\hline \multirow[t]{2}{*}{ Compound } & \multicolumn{2}{|c|}{$\%$ change in heart rate } & \multirow{2}{*}{$\begin{array}{l}\text { Decrease in the rats } \\
\text { arterial pressure }(\mathrm{mm} \mathrm{H})\end{array}$} \\
\hline & Decrease & Increase & \\
\hline 1 & - & 33.3 & 0 \\
\hline 2 & 0 & - & 0 \\
\hline 3 & - & 14.3 & 6.5 \\
\hline 4 & 22.7 & - & 2.6 \\
\hline 5 & 15 & - & 0 \\
\hline 6 & 0 & 40 & 0 \\
\hline 7 & 5.9 & - & 0 \\
\hline 8 & 14.3 & - & 0 \\
\hline 9 & 20 & - & 9.1 \\
\hline 10 & 5.9 & - & 0 \\
\hline 11 & 0 & - & 2.6 \\
\hline 12 & 16.7 & - & $2.6 \uparrow$ \\
\hline 13 & - & 8.3 & 0 \\
\hline 14 & - & 7.7 & 7.8 \\
\hline 15 & 10 & - & 5.2 \\
\hline 16 & 0 & - & 0 \\
\hline 17 & 5.9 & - & 6.5 \\
\hline 18 & 5.9 & - & 0 \\
\hline 19 & - & 28.5 & 6.5 \\
\hline
\end{tabular}

$\uparrow$ : Increase.

abolished in the tachycardia term and slightly induction on bradycardia was demonstrated by 7 . Similarly, transformation of $\mathbf{4}$ into $\mathbf{9}$ has emerged more bradycardia effect accomplished by moderate decreasing on blood pressure. However the product $\mathbf{8}$ showed induction on bradycardia and 10 exhibited only bradycardia effect. Further transformation of $\mathbf{1}$ into $\mathbf{1 1}$ does not seem to offer any advantages effect on heart rate but a very slightly decrease in blood pressure was observed. Whereas, compound 12 showed more bradycardia and completely abolished in tachycardia effects together with a slightly increase on blood pressure. Moreover, decreasing on tachycardia profile was exhibited by compound 13 . The replacement of the chlorine atom in $\mathbf{1 1}$ by ethoxide group gave 14, which has shown decrease on blood pressure and tachycardia effects. Thionation of $\mathbf{1}$ into 15 was accomplished by abolishing the tachycardia profile and induce on bradycardia term. The same behavior on tachycardia effect was observed by compound $\mathbf{1 6}$ but no influence on blood pressure profile has been recorded. Moreover, the transformation of $\mathbf{1 5}$ into $\mathbf{1 7}$ demonstrated almost the same behavior in the terms of the effects on heart rate and blood pressure. Whereas, compound $\mathbf{1 8}$ was exerted complete abolishing in tachycardia and slight induction on bradycardia profiles. However, induction on tachycardia and decreasing on blood pressure terms was demonstrated by compound 19 .

Structure modifications on the lead compounds 1-4 have afforded derivatives with different effects on heart rate and blood pressure profiles. Variation in the substituted alkyl groups has demonstrated slightly remarkable activity in regards to the parents, such as increasing on heart rate emerged by $\mathbf{6}$, induction in bradycardia proved by $\mathbf{5}$ and slightly decreasing in blood pressure occurred with $\mathbf{9}$. This could be attributed to the characteristic features of their alkyl groups. Introduction of lipophilic group on position 5 of the parent was appeared to offer slightly decrease in blood pressure in case of compounds $\mathbf{1 1}$ and $\mathbf{1 2}$. Nevertheless, the slight effect on blood pressure of $\mathbf{1 1}$ and 12 bearing at the 5 -position a chlorine atom indicates that, not only chloro lipophilic factor, but also the sterric effect are important for decrease on blood pressure as shown by 14. Thionation targets $\mathbf{1 5}$ and $\mathbf{1 6}$ showed remarkable attenuated on profiles activity, despite these compounds possess enhanced lipophilicity comparable to that parent compounds. However thioether products have almost emerged the same behavior on blood pressure and heart rate as in 17, and decreasing on blood pressure by 19 . The actual explanation for these changes regarding SAR will await the elucidation of the mechanism(s) of action of the compounds.

\section{Experimental \\ General}

Melting points were determined on open glass capillaries using a Mettler FP 62 apparatus and are uncorrected. The IR $\left(\mathrm{KBr}, \mathrm{v}, \mathrm{cm}^{-1}\right)$ spectra were recorded on a Perkin Elmer FT-IR Spectrum BX system. NMR spectra were recorded on a Bruker AMX 500 spectrometer in DMSO- $d_{6}$ and reported as $\delta$ ppm values relative to TMS at 500 and $125 \mathrm{MHz}$ for ${ }^{1} \mathrm{H}$ - and ${ }^{13} \mathrm{C}$ NMR, respectively. Mass spectra were measured on an Agilent 6410 TSQ system connected to Agilent 1200 HPLC interface (samples were infused in $\mathrm{MeOH}$ ). Follow up of the reactions and checking the purity of compounds was made by TLC on DC-Mikrokarten polygram SIL G/UV254, from the Macherey-Nagel Firm, Duren Thickness: $0.25 \mathrm{~mm}$. Column chromatography was conducted on silica gel (ICN Silica 100-200, active $60 \AA$ ).

\section{8-Methyl-2-phenoxy-4H-[1,2,4]triazolo[1,5-a]quinazolin-5-} one (3)

2-Hydrazino-5-methyl benzoic acid $(10 \mathrm{mmol})$ was added portion wise to a stirred solution of diphenyl$N$-cyanoimidocarabonate $(10 \mathrm{mmol})$ in $\mathrm{EtOH}(20 \mathrm{~mL})$ at $0^{\circ} \mathrm{C}$. Afterwards, triethylamine $(30 \mathrm{mmol})$ was added drop-wise over a period of $30 \mathrm{~min}$. After the addition was complete, the reaction mixture was left to stirr overnight at room temperature. Acidification of the mixture was 
performed by conc. $\mathrm{HCl}$ under ice cooling followed by refluxing for $1-3 \mathrm{~h}$. After cooling, the mixture was poured into ice/water, the resulting solid was filtered, washed with water and dried. Recrystallization from THF gave analytically pure colored as white solid, yield: $77 \%$, m.p. $218-220^{\circ} \mathrm{C}$; IR (KBr): $v / \mathrm{cm}^{-1} 1692$, $(\mathrm{C}=\mathrm{O}), 3219(\mathrm{NH}) ;{ }^{1} \mathrm{H}$ NMR (DMSO-d 6$): \delta=13.04$ (br s, 1H, NH), 7.98 (br s, $1 \mathrm{H}, \mathrm{H}-6), 7.72$ (br s, 2H, $\mathrm{H}-7,9), 7.45$ (td, $J=8.5,2 \mathrm{~Hz}, 2 \mathrm{H}, \mathrm{H}-3^{\prime} / 5^{\prime}$ ), 7.32 (br d, $J=8 \mathrm{~Hz}, 2 \mathrm{H}, \mathrm{H}-2^{\prime} / 6^{\prime}$ ), 7.25 (br t, $\left.J=7.5 \mathrm{~Hz}, 1 \mathrm{H}, \mathrm{H}-4^{\prime}\right)$, 2.45 (s, 3H, Ar- $\left.\mathrm{CH}_{3}\right) ;{ }^{13} \mathrm{C}$ NMR (DMSO- $\left.d_{6}\right): \delta=165.7$ (C-2), 159.4 (C-5), 154.2 (C-1'), 147.1 (C-9a), 136.2 (C-8), 135.3 (C-3a), 133.6 (C-5a), 129.7 (C-3'/5'), 127.8 (C-6), 124.9 (C-4'), 119.4 (C-2'/6'), 116.6 (C-7), 114.1 (C-9), 20.5 (Ar- $\left.\mathrm{CH}_{3}\right)$; MS (ESI) negative mode: $m / z 291.1\left(M^{-}-1\right)$ for $M W=292$.

2-Methylsulfonyl-4H-[1,2,4]triazolo[1,5-a]quinazolin-5-one (4) An amount of 2-methylsulfanyl-4H-[1,2,4]triazolo[1,5-a] quinazolin-5-one (1 mmol) was dissolved in bioling glacial acetic acid $(10 \mathrm{~mL})$, afterward $\mathrm{H}_{2} \mathrm{O}_{2}(10 \mathrm{~mL})$, was added drop-wise over a period of $5 \mathrm{~min}$. while heating. After the addition was complete, the mixture was poured into hot water and left at room temperature, the resulting solid was filtered, washed with water and dried. Recrystallization from toluene gave analytically pure colored as white solid, yield: $50 \%$, m.p. $261-263^{\circ} \mathrm{C}$; IR (KBr): $v / \mathrm{cm}^{-1} 1690(\mathrm{C}=\mathrm{O})$ $3240(\mathrm{NH}) ;{ }^{1} \mathrm{H}$ NMR (DMSO- $\left.d_{6}\right): \delta=13.39$ (br s, $1 \mathrm{H}, \mathrm{NH}$ ), 8.22 (br d, $J=8 \mathrm{~Hz}, 1 \mathrm{H}, \mathrm{H}-9$ ), 8.04 (br d, $J=8 \mathrm{~Hz}, 1 \mathrm{H}, \mathrm{H}-6$ ), 7.97 (br t, $J=7.5 \mathrm{~Hz}, 1 \mathrm{H}, \mathrm{H}-8$ ), 7.65 (br t, $J=7.5 \mathrm{~Hz}, 1 \mathrm{H}$, $\mathrm{H}-7$ ), 3.35 (s, 3H, $\left.\mathrm{O}_{2} S-\mathrm{CH}_{3}\right) ;{ }^{13} \mathrm{C} \mathrm{NMR}$ (DMSO-d $d_{6}$ ): $\delta=160.9$ (C-2), 160.2 (C-5), 149.9 (C-9a), 135.4 (C-8), 135.3 (C-3a), 128.4 (C-6), 127.2 (C-5a), 118.0 (C-7), 114.8 (C-9), $42.0\left(\mathrm{O}_{2} \mathrm{~S}-\mathrm{CH}_{3}\right) ; \mathrm{MS}$ (ESI) (negative mode): $\mathrm{m} / z$ $263.1\left(\mathrm{M}^{-}-1\right)$, (positive mode): $m / z 265.0\left(\mathrm{M}^{+}+\mathrm{H}\right), 287.1$ $\left(\mathrm{M}^{+}+\mathrm{Na}\right)$ for $\mathrm{MW}=264$.

\section{General procedure for synthesis of 2-phenoxy (methylsulfonyl)- 4-alkyl(aralkyl)-[1,2,4]-triazolo[1,5-a]quinazolin-5-ones (5-10)}

To a solution of $\mathbf{1}$ or $\mathbf{4}$ ( $\mathrm{I} \mathrm{mmol})$ in DMF $(5 \mathrm{~mL})$ was added potassium carbonate $(1.2 \mathrm{mmol})$ portion wise over a period of $10 \mathrm{~min}$ at room temperature. After stirring for $20 \mathrm{~min}$, the appropriate alkyl halide $(1.5 \mathrm{mmol})$ was added drop wise, and the reaction mixture was stirred for $18 \mathrm{~h}$ at room temperature. The mixture was poured into ice/water, the precipitate was filtered off, washed with water and dried. Analytically pure products 5-10 were obtained after recrystallization from THF.

\section{2-Phenoxy-4-(p-nitrobenzyl)-[1,2,4]triazolo[1,5-a]quinazolin- 5-one (5)}

White solid, yield: $78 \%$, m.p. $190-192^{\circ} \mathrm{C}$; IR (KBr): $v / \mathrm{cm}^{-1}$ $1677(\mathrm{C}=\mathrm{O}) ;{ }^{1} \mathrm{H}$ NMR (DMSO- $\left.d_{6}\right): \delta=8.25$ (dd, $J=8$, $1 \mathrm{~Hz}, 1 \mathrm{H}, \mathrm{H}-9), 8.19$ (d, $\left.J=8.5 \mathrm{~Hz}, 2 \mathrm{H}, \mathrm{H}-33^{\prime \prime} / 5^{\prime \prime}\right), 7.95$ $(\mathrm{td}, J=8.5,1.5 \mathrm{~Hz}, 1 \mathrm{H}, \mathrm{H}-8), 7.87$ (br d, $J=8 \mathrm{~Hz}, 1 \mathrm{H}$, $\mathrm{H}-6), 7.71$ (d, $\left.J=8.5 \mathrm{~Hz}, 2 \mathrm{H}, \mathrm{H}-2^{\prime \prime} / 6^{\prime \prime}\right), 7.59$ (td, $J=8$, $1 \mathrm{~Hz}, 1 \mathrm{H}, \mathrm{H}-7), 7.46$ (td, $\left.J=8.5,2 \mathrm{~Hz}, 2 \mathrm{H}, \mathrm{H}-3^{\prime} / 5^{\prime}\right)$, 7.33 (br d, $J=8 \mathrm{~Hz}, 2 \mathrm{H}, \mathrm{H}-2^{\prime} / 6^{\prime}$ ), 7.26 (br t, $J=7.5 \mathrm{~Hz}$, $1 \mathrm{H}, \mathrm{H}-4^{\prime}$ ), 5.43 (s, 2H, $-\mathrm{CH}_{2}-\mathrm{Ar}$ ); ${ }^{13} \mathrm{C}$ NMR (DMSO- $d_{6}$ ): $\delta=165.2(\mathrm{C}-2), 158.7(\mathrm{C}-5), 154.1\left(\mathrm{C}-1^{\prime}\right), 148.4$ (C-9a), 146.9 (C-4"), 143.4 (C-1"), 135.8 (C-8), 135.3 (C-3a), $129.8\left(\mathrm{C}-3^{\prime} / 5^{\prime}\right), 128.8$ (C-2"/6"), 128.6 (C-6), 126.0 (C-5a), 124.9 (C-4'), $121.5\left(\mathrm{C}-3^{\prime \prime} / 5^{\prime \prime}\right), 119.0$ (C-2'/6'), 116.2 (C-7), 114.2 (C-9), $46.1\left(-\mathrm{CH}_{2}-\mathrm{Ar}\right) ; \mathrm{MS}(\mathrm{EI}): \mathrm{m} / z(\%)$ $413\left(\mathrm{M}^{\cdot+}, 92\right)$ for $\mathrm{MW}=413$.

4-Ethyl-2-phenoxy-[1,2,4]triazolo[1,5-a]quinazolin-5-one (6) White solid, yield: $73 \%$, m.p. $165-167^{\circ} \mathrm{C}$; IR (KBr): $v / \mathrm{cm}^{-1}$ $1675(\mathrm{C}=\mathrm{O}) ;{ }^{1} \mathrm{H}$ NMR (DMSO- $\left.d_{6}\right): \delta=8.23(\mathrm{dd}, J=8$, $1 \mathrm{~Hz}, 1 \mathrm{H}, \mathrm{H}-9), 7.92$ (td, $J=8.5,1.5 \mathrm{~Hz}, 1 \mathrm{H}, \mathrm{H}-8), 7.83$ (br d, $J=8 \mathrm{~Hz}, 1 \mathrm{H}, \mathrm{H}-6), 7.56(\mathrm{td}, J=8.5,1 \mathrm{~Hz}, 1 \mathrm{H}$, $\mathrm{H}-7), 7.46\left(\mathrm{td}, J=8.5,1 \mathrm{~Hz}, 2 \mathrm{H}, \mathrm{H}-3^{\prime} / 5^{\prime}\right), 7.35$ (dd, $J=8.5$, $1 \mathrm{~Hz}, 2 \mathrm{H}, \mathrm{H}-2^{\prime} / 6^{\prime}$ ), 7.27 (br t, $J=7.5 \mathrm{~Hz}, 1 \mathrm{H}, \mathrm{H}-4^{\prime}$ ), 4.15 (q, $\left.J=7 \mathrm{~Hz}, 2 \mathrm{H},-\mathrm{CH}_{2}-\mathrm{CH}_{3}\right), 1.29\left(\mathrm{t}, J=7 \mathrm{~Hz}, 3 \mathrm{H},-\mathrm{CH}_{2}-\right.$ $\left.\mathrm{CH}_{3}\right) ;{ }^{13} \mathrm{C}$ NMR (DMSO- $\left.d_{6}\right): \delta=165.4(\mathrm{C}-2), 158.3(\mathrm{C}-5)$, 154.2 (C-1'), 148.2 (C-9a), 135.4 (C-8), 135.0 (C-3a), 129.8 (C-3'/5'), 128.4 (C-6), 125.8 (C-5a), 124.9 (C-4'), 119.1 (C-2'/6'), 116.3 (C-7), 114.1 (C-9), $38.5\left(-\mathrm{CH}_{2}-\mathrm{CH}_{3}\right), 12.4$ $\left(-\mathrm{CH}_{2}-\mathrm{CH}_{3}\right)$; MS (EI): $m / z$ (\%) $306\left(\mathrm{M}^{\circ+}\right.$, 98) for $\mathrm{MW}=306$.

\section{4-Allyl-2-phenoxy-[1,2,4]triazolo[1,5-a]quinazolin-5-one (7)}

White solid, yield: $85 \%$, m.p. $133-135^{\circ} \mathrm{C}$; IR (KBr): $v / \mathrm{cm}^{-1}$ $1684(\mathrm{C}=\mathrm{O}) ;{ }^{1} \mathrm{H}$ NMR (DMSO- $\left.d_{6}\right): \delta=8.23(\mathrm{br} \mathrm{d}, J=8 \mathrm{~Hz}$, $1 \mathrm{H}, \mathrm{H}-9), 7.92$ (td, $J=8.5,1.5 \mathrm{~Hz}, 1 \mathrm{H}, \mathrm{H}-8), 7.83$ (br d, $J=8 \mathrm{~Hz}, 1 \mathrm{H}, \mathrm{H}-6), 7.57(\mathrm{td}, J=8.5,1 \mathrm{~Hz}, 1 \mathrm{H}, \mathrm{H}-7)$, $7.47\left(\mathrm{td}, J=8.5,1 \mathrm{~Hz}, 2 \mathrm{H}, \mathrm{H}-3^{\prime} / 5^{\prime}\right), 7.35(\mathrm{dd}, J=8.5$, $1 \mathrm{~Hz}, 2 \mathrm{H}, \mathrm{H}-2^{\prime} / 6^{\prime}$ ), 7.27 (br t, $J=7.5 \mathrm{~Hz}, 1 \mathrm{H}, \mathrm{H}-4^{\prime}$ ), 5.97 (m, 1H, H-2"), 5.24 (dd, $J=17.5,1.5 \mathrm{~Hz}, 1 \mathrm{H}, \mathrm{H}-3 \mathrm{a}^{\prime \prime}$ ), 5.19 (dd, $\left.J=10.5,1.5 \mathrm{~Hz}, 1 \mathrm{H}, \mathrm{H}-3 \mathrm{~b}^{\prime \prime}\right), 4.72$ (d, $J=5 \mathrm{~Hz}$, $\left.2 \mathrm{H}, \mathrm{H}-1^{\prime \prime}\right) ;{ }^{13} \mathrm{C}$ NMR (DMSO- $\left.d_{6}\right): \delta=165.4$ (C-2), 158.2 (C-5), 154.2 (C-1'), 148.2 (C-9a), 135.5 (C-8), 135.1 (C-3a), 131.2 (C-2"), $129.8\left(\mathrm{C}-3^{\prime} / 5^{\prime}\right), 128.5$ (C-6), 125.9 (C-5a), 124.9 (C-4'), 119.1 (C-2'/6'), 117.5 (C-3"), 116.1 (C-7), 114.1 (C-9), 45.2 (C-1"); MS (EI): $m / z(\%)$ $318\left(\mathrm{M}^{\bullet+}, 98\right)$ for $\mathrm{MW}=318$.

\section{4-Ethyl-2-methylsulfonyl-[1,2,4]triazolo[1,5-a]quinazolin-5- one (8)}

White solid, yield: $71 \%$, m.p. $187-189^{\circ} \mathrm{C}$; IR (KBr): $v / \mathrm{cm}^{-1}$ $1675(\mathrm{C}=\mathrm{O}) ;{ }^{1} \mathrm{H}$ NMR (DMSO- $\left.d_{6}\right): \delta=8.27$ (br d, $J=8 \mathrm{~Hz}$, $1 \mathrm{H}, \mathrm{H}-9$ ), 8.07 (br d, $J=8 \mathrm{~Hz}, 1 \mathrm{H}, \mathrm{H}-6$ ), 8.00 (br t, $J=7.5 \mathrm{~Hz}, 1 \mathrm{H}, \mathrm{H}-8$ ), 7.69 (br t, $J=7.5 \mathrm{~Hz}, 1 \mathrm{H}, \mathrm{H}-7$ ), 4.22 (q, $\left.J=6.5 \mathrm{~Hz}, 2 \mathrm{H},-\mathrm{CH}_{2}-\mathrm{CH}_{3}\right), 3.49\left(\mathrm{~s}, 3 \mathrm{H}, \mathrm{O}_{2} \mathrm{~S}-\mathrm{CH}_{3}\right.$ ), $1.34\left(\mathrm{t}, J=6.5 \mathrm{~Hz}, 3 \mathrm{H},-\mathrm{CH}_{2}-\mathrm{CH}_{3}\right) ;{ }^{13} \mathrm{C}$ NMR (DMSO- $\left.d_{6}\right)$ : $\delta=160.8$ (C-2), 158.3 (C-5), 149.8 (C-9a), 135.5 (C-8), 134.5 (C-3a), 128.6 (C-6), 127.6 (C-5a), 117.4 (C-7), 114.8 
(C-9), $42.1\left(\mathrm{O}_{2} \mathrm{~S}-\mathrm{CH}_{3}\right), 38.9\left(-\mathrm{CH}_{2}-\mathrm{CH}_{3}\right), 12.4\left(-\mathrm{CH}_{2}-\mathrm{CH}_{3}\right)$; MS (EI): $m / z(\%) 292\left(M^{\bullet+}, 89\right)$ for MW = 292.

\section{4-Allyl-2-methylsulfonyl-[1,2,4]triazolo[1,5-a]quinazolin-5- one (9)}

White solid, yield: $87 \%$, m.p. $152-154^{\circ} \mathrm{C}$; IR (KBr): $v / \mathrm{cm}^{-1}$ 1684 $(\mathrm{C}=\mathrm{O}) ;{ }^{1} \mathrm{H}$ NMR (DMSO- $\left.d_{6}\right): \delta=8.27$ (br d, $J=8 \mathrm{~Hz}$, $1 \mathrm{H}, \mathrm{H}-9$ ), 8.10 (br d, $J=8 \mathrm{~Hz}, 1 \mathrm{H}, \mathrm{H}-6$ ), 8.03 (br t, $J=7.5 \mathrm{~Hz}$, $1 \mathrm{H}, \mathrm{H}-8$ ), 7.70 (br t, $J=7.5 \mathrm{~Hz}, 1 \mathrm{H}, \mathrm{H}-7), 6.00$ (m, 1H, H-2'), 5.31 (br d, $J=17.5 \mathrm{~Hz}, 1 \mathrm{H}, \mathrm{H}-3 \mathrm{a}^{\prime}$ ), 5.21 (br d, $J=10.5 \mathrm{~Hz}$, $\left.1 \mathrm{H}, \mathrm{H}-3 \mathrm{~b}^{\prime}\right), 4.80$ (d, J=5 Hz, 2H, H-1'), 3.48 (s, 3H, $\left.\mathrm{O}_{2} \mathrm{~S}-\mathrm{CH}_{3}\right) ;{ }^{13} \mathrm{C} \mathrm{NMR}$ (DMSO-d $\left.{ }_{6}\right): \delta=160.7(\mathrm{C}-2)$, 158.3 (C-5), 149.9 (C-9a), 135.7 (C-8), 134.8 (C-3a), 131.0 (C-2'), 128.7 (C-6), 127.7 (C-5a), 117.6 (C-3'), 117.2 (C-7), 114.9 (C-9), $45.5\left(\mathrm{C}-1^{\prime}\right), 42.0\left(\mathrm{O}_{2} \mathrm{~S}-\mathrm{CH}_{3}\right)$; MS (EI): $m / z(\%) 304\left(\mathrm{M}^{*+}, 100\right)$ for $\mathrm{MW}=304$.

\section{4-(p-Nitrobenzyl)-2-methylsulfonyl-[1,2,4]triazolo[1,5-a] quinazolin-5-one (10)}

White solid, yield: $72 \%$ m.p. $199-201^{\circ} \mathrm{C}$; IR (KBr): $v / \mathrm{cm}^{-1} 1678(\mathrm{C}=\mathrm{O}) ;{ }^{1} \mathrm{H}$ NMR (DMSO- $\left.d_{6}\right): \delta=8.29$ (br d, $J=8 \mathrm{~Hz}, 1 \mathrm{H}, \mathrm{H}-9), 8.19$ (d, $\left.J=8.5 \mathrm{~Hz}, 2 \mathrm{H}, \mathrm{H}-3^{\prime} / 5^{\prime}\right), 8.14$ (br d, $J=8 \mathrm{~Hz}, 1 \mathrm{H}, \mathrm{H}-6$ ), 8.05 (br t, $J=7.5 \mathrm{~Hz}, 1 \mathrm{H}, \mathrm{H}-8$ ), 7.75 (d, J=8.5 Hz, 2H, H-2"/6"), 7.72 (br t, $J=7.5 \mathrm{~Hz}, 1 \mathrm{H}$, $\mathrm{H}-7$ ), 5.51 (s, 2H, $-\mathrm{CH}_{2}$ - Ar), 3.47 (s, $3 \mathrm{H}, \mathrm{O}_{2} \mathrm{~S}-\mathrm{CH}_{3}$ ); ${ }^{13} \mathrm{C}$ NMR (DMSO- $d_{6}$ ): $\delta=160.6$ (C-2), 158.8 (C-5), 150.2 (C-9a), 146.9 (C-4'), 143.2 (C-1'), 135.9 (C-8), 135.0 (C-3a), 128.7 (C-2'/6'), 128.7 (C-6), 127.7 (C-5a), 123.5 (C-3'/5'), 117.3 (C-7), 114.9 (C-9), $46.5\left(-\mathrm{CH}_{2}-\right.$ Ar), $42.0\left(\mathrm{O}_{2} \mathrm{~S}-\mathrm{CH}_{3}\right)$; MS (EI): $m / z(\%) 399\left(\mathrm{M}^{\bullet+}, 90\right)$ for $\mathrm{MW}=399$.

\section{5-Chloro-2-phenoxy-[1,2,4]triazolo[1,5-a]quinazoline (11)}

Compound 1 (1 mmol) was refluxed with Phosphorous oxychloride $(1 \mathrm{~mL})$ in benzene $(7 \mathrm{~mL})$ for $2.5 \mathrm{~h}$. The solvent was evaporated and the residue was treated with saturated solution of potassium carbonate. The solid was filtered, washed thoroughly with water, dried and recrystallized from THF to give pure compound as white solid, yield: $82 \%$, m.p. 167-169 ${ }^{\circ}$; ${ }^{1} \mathrm{H}$ NMR (DMSO- $d_{6}$ ): $\delta=8.07$ (br d, $J=8 \mathrm{~Hz}$, $1 \mathrm{H}, \mathrm{H}-9), 7.64(\mathrm{~m}, 2 \mathrm{H}, \mathrm{H}-6 / 8), 7.40(\mathrm{td}, J=8.5,1 \mathrm{~Hz}, 2 \mathrm{H}$, $\left.\mathrm{H}-3^{\prime} / 5^{\prime}\right), 7.30(\mathrm{td}, J=8.5,1 \mathrm{~Hz}, 1 \mathrm{H}, \mathrm{H}-7), 7.25$ (dd, $J=8.5$, $\left.1 \mathrm{~Hz}, 2 \mathrm{H}, \mathrm{H}-2^{\prime} / 6^{\prime}\right), 7.18$ (br t, $\left.J=8 \mathrm{~Hz}, 1 \mathrm{H}, \mathrm{H}-4^{\prime}\right)$; ${ }^{13} \mathrm{C}$ NMR (DMSO- $\left.d_{6}\right): \delta=167.6$ (C-2), $166.3(\mathrm{C}-5)$, 157.0 (C-9a), 155.0 (C-1'), 136.3 (C-3a), 131.7 (C-8), $129.4\left(\mathrm{C}-3^{\prime} / 5^{\prime}\right), 128.0$ (C-7), 123.8 (C-4'), 123.0 (C-6), 119.0 (C-2'/6'), 118.6 (C-5a), 112.9 (C-9). MS (EI): $m / z$ (\%) $296\left(\mathrm{M}^{\bullet+}, 100\right)$ for $\mathrm{MW}=296$.

5-Chloro-2-methylsulfonyl-[1,2,4]triazolo[1,5-a]quinazoline (13) $1 \mathrm{mmol}$ of 2-methylsulfanyl-5-chloro-[1,2,4] triazolo[1,5a]quinazoline was dissolved in bioling glacial acetic acid (10 mL), afterward $\mathrm{H}_{2} \mathrm{O}_{2}(10 \mathrm{~mL})$ was added drop-wise over a period of $5 \mathrm{~min}$. while heating. After the addition was complete, the mixture was poured into hot water and lef at room temperature, the resulting solid was filtered, washed with water and dried. Recrystallization from Toluene gave analytically pure colored as white solid, yield: 62\%, m.p. $196-198^{\circ} \mathrm{C} ;{ }^{1} \mathrm{H}$ NMR (DMSO- $d_{6}$ ): $\delta=8.21$ (br d, $J=8 \mathrm{~Hz}, 1 \mathrm{H}, \mathrm{H}-9), 7.99$ (m, 2H, H-6/8), 7.66 (br t, $J=7 \mathrm{~Hz}$, $1 \mathrm{H}, \mathrm{H}-7), 3.54$ (s, 3H, $\left.\mathrm{O}_{2} \mathrm{~S}-\mathrm{CH}_{3}\right) ;{ }^{13} \mathrm{C} \mathrm{NMR} \mathrm{(DMSO-d} 6$ ): $\delta=162.9$ (C-5), 160.9 (C-2), 158.6 (C-9a), 137.3 (C-3a), 135.5 (C-8), 128.8 (C-7), 127.4 (C-6), 118.5 (C-5a), 114.7 (C-9), $42.1\left(\mathrm{O}_{2} \mathrm{~S}-\mathrm{CH}_{3}\right)$; MS (EI): $m / z(\%) 282\left(\mathrm{M}^{\bullet+}, 100\right)$ for $M W=282$.

\section{5-Ethoxy-2-phenoxy-[1,2,4]triazolo[1,5-a]quinazoline (14)}

A freshly prepared sodium ethoxide solution from sodium (150 mg) and absolut ethanol $(35 \mathrm{~mL})$ was reacted with compound 11 ( $1 \mathrm{mmol}$ ) by stirring at room temperature for $30 \mathrm{~min}$. Afterwards the solid was collected by filtration, air dried, and recrystallized from THF to give product as white solid, yield: $56 \%$, m.p. $197-199^{\circ} \mathrm{C}$; IR (KBr): $v / \mathrm{cm}^{-1}$ $1602(\mathrm{C}=\mathrm{N})$; ${ }^{1} \mathrm{H}$ NMR (DMSO- $\left.d_{6}\right): \delta=8.15$ (br d, $J=8 \mathrm{~Hz}$, $1 \mathrm{H}, \mathrm{H}-9$ ), 7.71 (br t, $J=7.5 \mathrm{~Hz}, 1 \mathrm{H}, \mathrm{H}-8$ ), 7.65 (br d, $J=8 \mathrm{~Hz}$, $1 \mathrm{H}, \mathrm{H}-6), 7.49$ (dt, $J=8.5,1 \mathrm{~Hz}, 2 \mathrm{H}, \mathrm{H}-3^{\prime} / 5^{\prime}$ ), 7.43 (br t, $J=7.5 \mathrm{~Hz}, 1 \mathrm{H}, \mathrm{H}-7), 7.36$ (dd, $\left.J=8.5,1 \mathrm{~Hz}, 2 \mathrm{H}, \mathrm{H}-2^{\prime} / 6^{\prime}\right)$, 7.04 (br t, $J=8 \mathrm{~Hz}, 1 \mathrm{H}, \mathrm{H}-4{ }^{\prime}$ ), 3.72 (q, $J=7 \mathrm{~Hz}, 2 \mathrm{H}, \mathrm{CH}_{2}$ ), 1.57 (t, $\left.J=7 \mathrm{~Hz}, 3 \mathrm{H}, \mathrm{CH}_{3}\right) ;{ }^{13} \mathrm{C}$ NMR (DMSO-d $): \delta=167.3$ (C-2), 155.5, (C-5), 154.2 (C-9a), 150.7 (C-1'), 135.6 (C-3a), 133.5 (C-8), $129.8\left(\mathrm{C}-3^{\prime} / 5^{\prime}\right), 127.9$ (C-7), 123.8 (C-4'), 123.0 (C-6), 119.6 (C-2'/6'), 118.9 (C-5a), 112.9 (C-9), 24.1 $\left(-\mathrm{CH}_{2} \mathrm{CH}_{3}\right), 13.9\left(-\mathrm{CH}_{2} \mathrm{CH}_{3}\right) ; \mathrm{MS}(\mathrm{EI}): m / z$ (\%) 306 $\left(\mathrm{M}^{\bullet+}, 73\right)$ for $\mathrm{MW}=306$.

\section{2-Phenoxy-4H-[1,2,4]triazolo[1,5-a]quinazolin-5-thione (15)} Compounds 1 ( $1 \mathrm{mmol}$ ) was refluxed with phosphorous pentasulfide $(1 \mathrm{mmol})$ in absolute pyridine $(5 \mathrm{~mL})$ for $3 \mathrm{~h}$. Afterwards the reaction mixture was cooled and poured into ice/water, the yellow precipitate was separated by filtration and washed thoroughly with water. Recrystallization from aqueous dimethylformamide furnished analytically pure as yellow solid, yield: $92 \%$, m.p. $209-211^{\circ} \mathrm{C}$; IR (KBr): $v / \mathrm{cm}^{-1} 1197(\mathrm{C}=\mathrm{S}) ;{ }^{1} \mathrm{H}$ NMR $\left(\mathrm{DMSO}-d_{6}\right): \delta=8.64$ (dd, $J=8,1 \mathrm{~Hz}, 1 \mathrm{H}, \mathrm{H}-9), 7.95$ (br dt, $J=7.5,1 \mathrm{~Hz}$, $1 \mathrm{H}, \mathrm{H}-8$ ), 7.89 (br d, $J=7.5 \mathrm{~Hz}, 1 \mathrm{H}, \mathrm{H}-6), 7.56$ (td, $J=8$, $1 \mathrm{~Hz}, 1 \mathrm{H}, \mathrm{H}-7), 7.47$ (td, $\left.J=8.5,1 \mathrm{~Hz}, 2 \mathrm{H}, \mathrm{H}-3^{\prime} / 5^{\prime}\right), 7.35$ (dd, $J=8.5,1 \mathrm{~Hz}, 2 \mathrm{H}, \mathrm{H}-2^{\prime} / 6^{\prime}$ ), 7.27 (br t, $J=7.5 \mathrm{~Hz}, 1 \mathrm{H}$, H-4' $) ;{ }^{13} \mathrm{C}$ NMR (DMSO- $d_{6}$ ): $\delta=185.3$ (C-5), 165.0 (C-2), 154.2 (C-1'), 149.3 (C-9a), 135.9 (C-8),132.4 (C-3a), 131.8 (C-5a), 129.8 (C-3'/5'), 126.3 (C-6), 125.0 (C-4'), 123.9 (C-7), 119.4 (C-2'/6'), 114.5 (C-9); MS (EI): $m / z$ (\%) 294 $\left(\mathrm{M}^{+}, 90\right)$ for $\mathrm{MW}=294$.

\section{General procedure for synthesis of 5-Alkyl(phenyl)sulfanyl- 2-phenxy-[1,2,4]triazolo[1,5-a]-quinazolines (17-19)}

Compound 15 ( $1 \mathrm{mmol})$ was dissolved in $2 \mathrm{M}$ sodium hydroxide solution $(10 \mathrm{~mL})$, alkyl halide $(1.5 \mathrm{mmol})$ was 
added dropwise over a period $2 \mathrm{~min}$, the mixture was left to stir for $\mathrm{I} h$ at room temperature, and the obtained solid was separated by filtration, washed thoroughly with water and dried. Recrystallization of the crude products from THF afforded 17-19 as colored pure solid.

5-Ethylsulfanyl-2-phenoxy-[1,2,4]triazolo[1,5-a]quinazoline (17) White solid, yield: $68 \%$, m.p. $185-187^{\circ} \mathrm{C} ;{ }^{1} \mathrm{H}$ NMR (DMSO- $d_{6}$ ): $\delta=8.26$ (br d, $J=8 \mathrm{~Hz}, 1 \mathrm{H}, \mathrm{H}-9$ ), 7.75 (br t, $J=7.5 \mathrm{~Hz}, 1 \mathrm{H}, \mathrm{H}-8$ ), 7.77 (br d, $J=8 \mathrm{~Hz}, 1 \mathrm{H}, \mathrm{H}-6$ ), 7.63 (dt, $J=8.5,1 \mathrm{~Hz}, 2 \mathrm{H}, \mathrm{H}-3^{\prime} / 5^{\prime}$ ), 7.51 (br t, $J=7.5 \mathrm{~Hz}, 1 \mathrm{H}$, $\mathrm{H}-7$ ), 7.41 (dd, $J=8.5,1 \mathrm{~Hz}, 2 \mathrm{H}, \mathrm{H}-2^{\prime} / 6^{\prime}$ ), 7.11 (br t, $\left.J=8 \mathrm{~Hz}, 1 \mathrm{H}, \mathrm{H}-4^{\prime}\right), 4.39$ (q, J=7.2 Hz, 2H, $\mathrm{CH}_{2}$ ), $1.41\left(\mathrm{t}, J=7.2 \mathrm{~Hz}, 3 \mathrm{H}, \mathrm{CH}_{3}\right) ;{ }^{13} \mathrm{C} \mathrm{NMR}$ (DMSO- $\left.d_{6}\right)$ : $\delta=167.9(\mathrm{C}-2), 156.7,(\mathrm{C}-5), 154.0(\mathrm{C}-9 \mathrm{a}), 150.9\left(\mathrm{C}-1^{\prime}\right)$, 136.1 (C-3a),134.8 (C-8), 130.1 (C-3'/5'), 128.3 (C-7), 125.2 (C-4'), 123.9 (C-6), 120.3 (C-2'/6'), 119.1 (C-5a), 112.1 (C-9), $62.1\left(-\mathrm{CH}_{2} \mathrm{CH}_{3}\right), 13.9\left(-\mathrm{CH}_{2} \mathrm{CH}_{3}\right)$; $\mathrm{MS}$ (EI): $m / z(\%) 322\left(\mathrm{M}^{\bullet+}, 72\right)$ for $\mathrm{MW}=322$.

\section{5-Allylsulfanyl-2-phenoxy-[1,2,4]triazolo[1,5-a]quinazoline (18)}

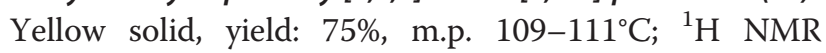
(DMSO- $d_{6}$ ): $\delta=8.08$ (br d, $J=8 \mathrm{~Hz}, 1 \mathrm{H}, \mathrm{H}-9$ ), 7.72 (td, $J=8.5,1.5 \mathrm{~Hz}, 1 \mathrm{H}, \mathrm{H}-8), 7.66(\mathrm{br} \mathrm{d}, J=8 \mathrm{~Hz}, 1 \mathrm{H}$, H-6), 7.37 (td, $\left.J=8.5,1 \mathrm{~Hz}, 2 \mathrm{H}, \mathrm{H}-3^{\prime} / 5^{\prime}\right), 7.33(\mathrm{td}, J=8.5$, $1 \mathrm{~Hz}, 1 \mathrm{H}, \mathrm{H}-7), 7.25$ (dd, $\left.J=8.5,1 \mathrm{~Hz}, 2 \mathrm{H}, \mathrm{H}-2^{\prime} / 6^{\prime}\right), 7.17$ (br t, J=7.5 Hz, 1H, H-4'), 6.04 (m, 1H, H-2"), 5.42 (dd, $J=17.5,1.5 \mathrm{~Hz}, 1 \mathrm{H}, \mathrm{H}-3 \mathrm{a}^{\prime \prime}$ ), 5.20 (dd, $J=10.5$, $\left.1.5 \mathrm{~Hz}, 1 \mathrm{H}, \mathrm{H}-3 \mathrm{~b}^{\prime \prime}\right), 4.09$ (d, J=6.5 Hz, $\left.2 \mathrm{H}, \mathrm{H}-1^{\prime \prime}\right) ;{ }^{13} \mathrm{C}$ NMR (DMSO- $d_{6}$ ): $\delta=167.4$ (C-2), 166.6 (C-5), 154.9 (C-1'), 154.2 (C-5a), 151.0 (C-9a), 136.2 (C-3a), 135.7 (C-8), 129.6 (C-2'), 129.5 (C-3'/5'), 127.9 (C-6), 125.0 (C-7), 123.9 (C-4'), 119.0 (C-2'/6'), 115.1 (C-3"), 112.9 (C-9), 32.1 (C-1"); MS (EI): $m / z(\%) 334\left(\mathrm{M}^{{ }^{+}}, 89\right)$ for $\mathrm{MW}=334$.

\section{2-Phenoxy-5-phenylsulfanyl-[1,2,4]triazolo[1,5-a]quinazoline (19)} Yellow solid, yield: $54 \%$, m.p. $172-174^{\circ} \mathrm{C} ;{ }^{1} \mathrm{H}$ NMR (DMSO- $d_{6}$ ): $\delta=8.81$ (br dd, $J=8.5,1 \mathrm{~Hz}, 1 \mathrm{H}, \mathrm{H}-9$ ), 7.78 (br d, $J=8 \mathrm{~Hz}, 1 \mathrm{H}, \mathrm{H}-6), 7.71$ (td, $J=8.5,1.5 \mathrm{~Hz}, 1 \mathrm{H}, \mathrm{H}-8)$, 7.66 (td, $J=8.5,1 \mathrm{~Hz}, 1 \mathrm{H}, \mathrm{H}-7), 7.43(\mathrm{td}, J=8.5,1 \mathrm{~Hz}, 2 \mathrm{H}$, $\left.\mathrm{H}-3^{\prime} / 5^{\prime}\right), 7.38\left(\mathrm{td}, J=8.5,1 \mathrm{~Hz}, 2 \mathrm{H}, \mathrm{H}-3^{\prime \prime} / 5^{\prime \prime}\right), 7.31$ $\left(\mathrm{dd}, J=8.5,1 \mathrm{~Hz}, 2 \mathrm{H}, \mathrm{H}-2^{\prime} / 6^{\prime}\right), 7.27$ (dd, $J=8.5$, $\left.1 \mathrm{~Hz}, 2 \mathrm{H}, \mathrm{H}-2^{\prime \prime} / 6^{\prime \prime}\right), 7.21$ (br t, $J=8 \mathrm{~Hz}, 1 \mathrm{H}, \mathrm{H}-4^{\prime}$ ), 7.17 (br t, $\left.J=7.5 \mathrm{~Hz}, 1 \mathrm{H}, \mathrm{H}-4{ }^{\prime \prime}\right) ;{ }^{13} \mathrm{C}$ NMR (DMSO- $d_{6}$ ): $\delta=166.8$ (C-2), 155.0 (C-5), 154.8 (C-1'), 152.4 (C-3a), 132.9 (C-9a), 132.3 (C-8), 132.1 (C-3"/5"), 129.5 (C-3'/5', 1"), 129.4 (C-4"), 127.9 (C-5a), 124.2 (C-4',2"/6"), 123.8 (C-6), (C-3'/5'), $119.1\left(\mathrm{C}-2^{\prime} / 6^{\prime}\right), 119.0$ (C-7), 113.5 (C-9); MS (EI): $m / z(\%) 370\left(\mathrm{M}^{+}, 74\right)$ for $\mathrm{MW}=370$.

\section{Antihypertensive activity}

In vivo antihypertensive activity study of the title compounds was performed by tail cuff method using Blood Pressure Monitor for rats and mice (Model MK 2000- Muromachi kikkai Co. ltd. Japan). The samples of the investigated compounds were administered orally at a dose of $5 \mathrm{mg} / \mathrm{kg}$ as suspension in 1\% sodium carboxy methyl cellulose. Measurements (blood pressure and heart rate) were recorded before and after the treatment of tested compounds at the intervals of $1 \mathrm{~h}$ for $5 \mathrm{~h}$.

\section{Conclusions}

Since compounds 4, 8, 9, 12, and 15 have abolished completely the tachycardia of the parent compounds, they may be studied as potential adrenoblockers. Compounds 9 and $\mathbf{1 4}$ may be modified to enhance their hypotensive activity. Furthermore, compound $\mathbf{6}$ seem to be a cardiac stimulant and it will be studied further for this concern. Finally, the structure-activity relationship (SAR) study of the compounds gave us some useful insights about the characteristic requirements, which may be taken into consideration in the design of new antihypertensive agents.

\section{Competing interests}

The authors declare that they have no competing interests.

\section{Authors' contributions}

RA and NL have made a substantial contribution to experimental design. RA and MM made a significant contribution to acquisition of data, analysis, manuscript preparation. KE designed and performed the biological study and manuscript revision. IA and $\mathrm{MH}$ read, revised and approved the final manuscript. All authors read and approved the final manuscript.

\section{Acknowledgement}

The authors extend their appreciation to the Deanship of Scientific Research at King Saud University for funding this work through research group No RGP-VPP-291.

\section{Author details}

'Department of Pharmacetical Chemistry, College of Pharmacy, King Saud University, P. O. Box 2457, Riyadh 11451, Saudi Arabia. ${ }^{2}$ Department of Pharmacology, College of Pharmacy, King Saud University, P. O. Box 2457, Riyadh 11451, Saudi Arabia. ${ }^{3}$ Department of Pharmaceutical Chemistry, College of Pharmacy, Aleppo University, Aleppo, Syria.

Received: 16 September 2013 Accepted: 13 January 2014 Published: 15 January 2014

\section{References}

1. Alagarsamy $V$, Pathak US: Synthesis and antihypertensive activity of novel 3-benzyl-2-substituted $3 \mathrm{H}$-[1,2,4]triazolo[5,1-b]quinazolin-9-ones. Bioorg Med Chem 2007, 15:3457-3462.

2. Garcia DJ, Somanathan R, Rivero IA, Aguirre G, Hellberg LH: Synthesis of deuterium-labeled antihypertensive 3-(4-Phenyl-1'-Piperazinyl)-Propyl-2,4quinazolinedione. Synth Commun 2000, 30:2707-2711.

3. Alagarsamy V, Venkatesaperumal R, Vijayakumar S, Angayarkanni T, Pounammal P, Senthilganesh S, Kandeeban S: Synthesis and pharmacological investigation of some novel 2-phenyl-3-(substituted methyl amino) quinazolin-4(3 h)-ones as $h_{1}$-receptor blockers. Pharmazie 2002, 57:306-307.

4. Alagarsamy $V$ : Synthesis and pharmacological investigation of some novel 2-methyl-3-(substituted methylamino)-(3H)-quinazolin-4-ones as histamine H1 receptor blockers. Pharmazie 2004, 59:753-755. 
5. Alagarsamy V, Solomon VR, Vanikavitha G, Paluchamy V, Ravichandran M, Arnaldsujin A, Thangathirupathy A, Amuthalakshmi S, Revathi R: Synthesis, analgesic, anti-inflammatory and antibacterial activities of some novel 2-phenyl-3-substituted quinazolin-4(3H) ones. Biol Pharm Bull 2002, 25:1432-1435.

6. Alagarsamy V, Murugananthan G, Venkateshperumal R: Synthesis, analgesic, anti-inflammatory and antibacterial activities of some novel 2-methyl-3-substituted quinazolin-4-(3H)-ones. Biol Pharm Bull 2003, 26:1711-1714.

7. MannJen H, Jiau HL, Sheng Chu K, Yi Bastow X, Kenneth B, Hamel NY, Lee E, Hsiung KJ: 6-Alkylamino- and 2,3-dihydro-3'-methoxy-2-phenyl-4quinazolinones and related compounds: their synthesis, cytotoxicity, and inhibition of tubulin polymerization. J Med Chem 2000, 43:4479-4487.

8. Alagarsamy V, Revathi R, Meena S, Ramaseshu KV, Rajasekaran S, De Clercq E: AntiHIV, antibacterial and antifungal activities of some 2,3-disubstituted quinazolin-4(3H)-ones. Indian J Pharm Sci 2004, 66:459-462.

9. Al-Salahi R, Marzouk M, Awad G, Al-Omar M, Ezzeldin E: Antimicrobial activity of a newly synthesized 2-methylsulfanyl-[1,2,4]triazolo[1,5-a]quinazolin-5-one and its derivatives. J Pharm Pharmacol 2013, 65:790-797.

10. Al-Salahi R, Al-Omar M, Alswaidan I, Marzouk M, Alsenousy W, Amr A: Antiviral activities of some methylsulfanyltriazoloquinazolines. Res Chem Intermed 2013. In press.

11. Al-Salahi R, Geffken D, Koellner M: A new series of 2-alkoxy(aralkoxy)$[1,2,4]$ triazolo[1,5-a]quinazolin-5-ones as adenosine receptor antagonists. Chem Pharm Bull 2011, 59:730-733.

12. Al-Salahi R, Marzouk M, Gamal Eldeen A: Cytotoxic and antiinflammatory active of a newly synthesized 2-methylsulfanyl-[1,2,4]triazolo[1,5-a]quinazolin5-one and its derivatives. J Pure Appl Microbiol 2013, 7:189-198.

13. Al-Salahi R, Geffken D: Synthesis of novel 2-alkoxy(aralkoxy)-4H-[1,2,4] triazolo[1,5-a]quinazolin-5-ones starting with dialkyl- $\mathrm{N}$ cyanoimidocarbonates. J Heterocycl Chem 2011, 48:656-662.

14. Al-Salahi R, Geffken D: Synthesis of 2-methylsulfanyl-4H-[1,2,4]triazolo[1,5a]quinazolin-5-one and derivatives. Synth Comm 2011, 41:3512-3523.

15. Lolak N: Synthesis of heterocyclic systems derived from diphenyl-Ncyanoimidocarbonate, Ph.D. Dissertation. Germany: Hamburg University; 2008:1-135.

16. Al-Salahi R, Lolak N, Al-Omar M, Ng SW: 2-phenoxy-4H-[1,2,4]triazolo[1,5-a] quinazolin-5-one. Acta Cryst 2012, E68:01808.

17. Al-Salahi R, Marzouk M, Al-Omar MA, Amr AE, Ng SW, Tiekink ERT 2-Methylsulfanyl-1,2,4-triazolo[1,5-a]quinazoline-5(4H)-thione. Acta Cryst 2013, E69:0434.

18. Al-Salahi R, Geffken D: Synthesis and reactivity of $[1,2,4]$ triazolo-annelated quinazolines. Molecules 2010, 15:7016-7034.

19. Al-Salahi R, Geffken D: Novel synthesis of 2-alkoxy(aralkoxy)-5-chloro $[1,2,4]$-triazolo[1,5-a]quinazoline and their derivatives. Heterocycles 2010, 81:1843-1859

20. Pfeiffer WD, Hetzheim A, Pazdera P, Bodtke A, Mueke J: Synthesis and reactivity of 1,2,4-triazolo-[1,5-c]quinazolines. J Hetrocyclic Chem 1999, 36:1327-1336

doi:10.1186/1752-153X-8-3

Cite this article as: Al-Salahi et al:: Biological effects of a new set 1,2,4-triazolo[1,5-a]quinazolines on heart rate and blood pressure. Chemistry Central Journal 2014 8:3.

Publish with ChemistryCentral and every
scientist can read your work free of charge
"Open access provides opportunities to our
colleagues in other parts of the globe, by allowing
anyone to view the content free of charge."
W. Jeffery Hurst, The Hershey Company.
- available free of charge to the entire scientific community
- peer reviewed and published immediately upon acceptance
- cited in PubMed and archived on PubMed Central
- yours - you keep the copyright
Submit your manuscript here:
http://www.chemistrycentral.com/manuscript/

